\title{
Non invasive optical cellular imaging in humans.
}

\author{
Maciej Wojtkowski, ${ }^{* 1,2}$, Małgorzata Jędrzejewska-Szczerska ${ }^{3}$, Dawid Borycki ${ }^{1}$ \\ ${ }^{1}$ Institute of Physical Chemistry, Polish Academy of Sciences, Kasprzaka Str. 44/52 01-224 Warszawa, \\ ${ }^{2}$ Institute of Physics, Faculty of Physics, Astronomy and Informatics, Nicolaus Copernicus University, ul. \\ Grudziadzka 5, 87-100 Torun, Poland \\ ${ }^{3}$ Faculty of Electronics, Telecommunications and Informatics, Gdańsk University of Technology, G. Narutowicza
} 11/12, 80-233 Gdańsk,

\begin{abstract}
One of the most appealing and still unsolved problems in biological and medical imaging is the possibility of noninvasive visualization of tissue in vivo with an accuracy of microscopic examination. A major difficulty to solve in biomedical imaging is a degradation of image quality caused by the presence of optical inhomogeneity of tissue. Is there any chance to develop a microscopic method that allows non-invasive observation of cells in living tissue?
\end{abstract}

Classical optical microscopy requires first to retrieve, then to process and finally to stain the sample, which is usually not possible to be performed in vivo. One of the possible ways to overcome limitations of classical microscopes is to introduce Confocal Microscopy, which allows axial sectioning and high resolution imaging of "optically extracted" slice of tissue without need of physical processing of imaged samples. This can be done by using spatially coherent beam (enabling full control of the beam shape) and a small pinhole placed in the front of the detector. However, there are two fundamental limitations of such configuration: confocal microscope has to operate at short working distances in order to get high resolution axial sectioning; high spatial coherence of illuminating beam causes that it is not possible to image objects which strongly scatter light. The first limitation can be bypassed by using temporal partially coherent light, which enables to apply time gating to improve axial sectioning. Unfortunately, this modality, called Optical Coherence Tomography (OCT) is not able to fix the second limitation of Confocal Microscopy. An alternative option is to use Autofluorescence Imaging (AI) that may help to avoid negative effects of strong scattering. Unfortunately, AI requires to deliver significant amount of energy - not always allowed for living organisms. Additionally, it does not allow to improve the axial sectioning like OCT. Confocal Microscopy, AI and OCT techniques can be also supported by Adaptive Optics (AO) methods. This modality enables to correct adaptively the wavefront aberrations introduced by slightly uneven distribution of the refractive index by measuring and categorizing wavefront distortions and

*E-mail: mwojtkowski@ichf.edu.pl correcting them using dedicated deformable mirrors. Classical adaptive optics (AO), with all its successful solutions to the field of astronomy, communication and ophthalmology, has no chance to succeed in healing multiple scattered beam. The reason is related to some fundamental restrictions: lack of high quality feedback beam for wavefront detector or inability of creation complex wavefront shapes of existing membrane mirrors based on well-established polynomial wavefront fitting. All of these techniques use spatially coherent illumination mainly for delivering high energy density to the sample enabling significant amount of light power to come back to a detection system. Some of them like OCT or other holographic techniques require additionally to use coherent radiation for having access to relative phase information. High spatial coherence of delivered light results in the presence of the speckle pattern preventing from seeing the smallest detectable details in the image. However, the speckle pattern encodes an information about light propagation. With proper complexity of experimental setup it is possible to treat the propagation of light through scattering media as deterministic process and describe in the classical regime where scattering objects are modeled as continuous 3D maps of refractive index. It has been already demonstrated that it is possible to reconstruct the amplitude and phase of light, which propagates through scattering medium and use this information to refocus light inside the scattering medium by digital optical phase conjugation.

Which method will be developed next? Are we already close to achieve the intended goal? Everything indicates that this is what is happening. The combination of new interferometric techniques such as OCT with advanced computational methods enabling numerical correction of aberration and purely optical cleaning of the effects of wavefront aberrations are beginning to give long-awaited effects. We can expect that in the next ten years there will be instruments to see cellular structures in the skin, eye and other internal organs that can be reached with an endoscope. 